\section{Animal welfare science aids conservation}

In their Policy Forum "Engage with animal welfare in conservation" (7 August, p. 629), N. Sekar and D. Shiller overlooked a long history of conservation-related animal welfare science. The integration of animal welfare science and conservation spans at least 60 years (1) and has been applied to a broad range of wildlife management activities (2) and interdisciplinary research (3-5). Understanding and incorporating animal welfare science can benefit conservation efforts.

Animal welfare science is not synonymous with opposition to intentional killing of wildlife (compassionate conservation) (6, 7). Rather, animal welfare science uses quantitative measurements to assess harmful and positive impacts of human activities on animals (8). Traditionally, the harms are weighed against conservation benefits to justify (or rule out) a management action. Approaches such as compassionate conservation may, perhaps counterintuitively, worsen animal welfare outcomes and make biodiversity conservation more difficult (9).

Sekar and Shiller use prescriptive advocacy framing that does not represent animal welfare science. Stipulating that conservation agencies should avoid factory farming products does not reflect scientific quantification and comparison of harms posed by this and other human activities. Processes such as land clearing (10) may pose greater animal welfare impacts when all wild sentient species and types of harms (11) are considered.

We agree that increased animal welfare focus is warranted in conservation. Progress will be expedited by wider collaboration with animal welfare scientists. Decades-old scientific tradition in this space teaches us to prioritize objective assessment of harms rather than deferring to advocacy.

Jordan O. Hampton ${ }^{1 *}$, Sandra E. Baker ${ }^{2}$, Ngaio J. Beausoleil', Marc Cattet ${ }^{4}$, David M. Forsyth ${ }^{5,6}$, Clive R. McMahon ${ }^{7}$, Gilbert Proulx ${ }^{8}$, Bruce Warburton $^{9}$

${ }^{1}$ School of Veterinary and Life Sciences, Murdoch University, Murdoch, WA 6150, Australia. ${ }^{2}$ Wildlife Conservation Research Unit, Department of Zoology, Recanati-Kaplan Centre, University of Oxford, OX13 5JL, UK. ${ }^{3}$ Animal Welfare Science and Bioethics Centre, School of Veterinary Science, Massey University, Palmerston North 4442, New Zealand. ${ }^{4}$ RGL Recovery Wildlife Health and Veterinary Services, Saskatoon, SK S7H 4A6, Canada. ${ }^{5}$ Vertebrate Pest Research Unit, Department of Primary Industries, Orange, NSW 2800, Australia. ${ }^{6} \mathrm{School}$ of Biological, Earth and Environmental Sciences, University of New South Wales, Sydney, NSW 2052, Australia. ${ }^{7}$ MOS Animal Satellite Tagging, Sydney Institute of Marine Science, Mosman, NSW 2088, Australia. ${ }^{8}$ Alpha Wildlife Research \& Management Ltd., Sherwood Park, AB T8H 1W3, Canada. ${ }^{9}$ Landcare Research, Lincoln 7640, New
*Corresponding author. Email: jordan.hampton@murdoch.edu.au

REFERENCES AND NOTES

1.B. Warburton, J. Hall, New Zealand J. Zool. 22, 39 (1995).

2.S. E. Baker et al., BioScience 63, 928 (2013).

3.D. Fraser, Anim. Welfare 19, 121 (2010).

4.B. Warburton, B. Norton, J. Wildl. Manag. 73, 158 (2009).

5.A. M. Harvey, N. J. Beausoleil, D. Ramp, D. J. Mellor, Animals 10, 148 (2020).

6.A. D. Wallach, M. Bekoff, C. Batavia, M. P. Nelson, D. Ramp, Conserv. Biol. 32, 1255 (2018).

7.N. J. Beausoleil, Animals 10, 257 (2020).

8.C. R. McMahon, M. A. Hindell, R. G. Harcourt, Wildl. Res. 39, 375 (2012).

9.A. Callen et al., Biol. Conserv. 241, 108365 (2020).

10.H. Finn, N. Stephens, WildI. Res. 44, 377 (2017).

11.D. Fraser, A. M. MacRae, Anim. Welfare 20, 581 (2011).

10.1126/science.abe2171 